\title{
Use of an autoperfusion catheter in the treatment of acute refractory vessel closure after coronary balloon angioplasty: immediate and six month follow up results
}

Johan M Van Lierde, James J Glazier, Francis J Stammen, Matty C Vrolix, Dimitris Sionis, Hilaire De Geest, Jan H Piessens

\begin{abstract}
Objective-To determine the usefulness of the Stack autopersion dilatation catheter in patients with acute recurrent vessel closure during coronary angioplasty.

Design-Prospective data collection.

Setting-University hospital.

Patients-In 37 of 1003 consecutive patients undergoing angioplasty between November 1989 and December 1990 acute vessel closure developed that could not be redilated by a conventional balloon catheter. $13(35 \%)$ of these 37 patients were sent immediately for emergency bypass surgery.
\end{abstract}

Intervention-In the remaining 24 patients an attempt was made to reopen the vessel with a Stack catheter.

Main outcome measure-Successful reopening of the vessel. All successfully treated patients were followed for at least six months to detect recurrent ischaemia.

Results-In 16 patients $(67 \%)$ the Stack procedure was successful. Of the eight patients in whom reopening of the occluded vessel was not achieved, seven were sent for bypass surgery and one was successfully treated by emergency stent implantation. The 16 patients successfully treated with the Stack autoperfusion system were followed up for a mean (SD) of $6.7(2.6)$ (range 2 to 11) months. Ten patients remained symptom free but early clinical restenosis developed in four (25\%). Overall, only three $(19 \%)$ of 16 patients experienced recurrence of severe (class III-IV) symptoms and required further mechanical revascularisation.

Conclusion-These data support the use of the Stack autoperfusion catheter system in selected patients with acute vessel closure not responsive to attempted redilatation with conventional balloon catheters. The short-term outcome seen in this series of patients who were successfully treated with this coronary autoperfusion system is encouraging.

Acute reocclusion remains a major problem during and after percutaneous transluminal coronary angioplasty. ${ }^{12}$ It is the main cause of post-procedural emergency surgery, myocardial infarction, and even death. Acute reocclusion is generally treated with conventional redilatation. Prolonged inflations are often attempted but the development of severe anginal pain, hypotension, or electrical instability often limits the inflation time. An autoperfusion catheter can be used to permit longer inflation times. ${ }^{34}$

We report our experience with the Stack autoperfusion balloon catheter in the treatment of patients with acute vessel closure after angioplasty in whom attempted redilatation with a conventional balloon catheter was unsuccessful.

\section{Patients and methods}

In the first year after the introduction of the Stack autoperfusion balloon catheter at our centre (in November 1989) a total of 1003 coronary angioplasties were performed. On our computerised angioplasty registry ${ }^{5}$ we identified all the patients (37) who developed persistent and acute vessel closure after attempted redilatation with a conventional balloon catheter. Thirteen of these 37 patients were sent immediately to bypass surgery because the angioplasty operator judged the underlying dissection to be so extensive and complex as to preclude success with an autoperfusion catheter system. In one of these 13 patients the left main stem was dissected by the guiding catheter, in eight there was an extensive dissection of the proximal left anterior descending artery including one or two major diagonal branches, and in the other four the occlusive dissection started proximal on a dominant right coronary artery and extended to the crux. In the remaining 24 patients we attempted to restore patency of the occluded vessel with a Stack autoperfusion balloon catheter system (Advanced Cardiovascular Systems, Temecula, California).

\section{CLINICAL AND ANGIOGRAPHIC DEFINITIONS}

Clinical data were collected from the angioplasty registry data base and the hospital records. The angina class was assigned according to the Canadian Cardiovascular Society Classification. ${ }^{6}$ Current cigarette smokers were defined as patients who admitted to smoking any amount regularly within three months of presentation. Arterial hypertension and
Accepted

19 September 1991

$\star$ Presented in part at the European Congress Cardiology, 1991, Netherlands. 
diabetes mellitus were defined as necessitating treatment. Family history of coronary artery disease was one that included parents or siblings. Lipid values were obtained after a 14 hour fast, and hypercholesterolaemia was defined as a fasting serum cholesterol concentration $>6.2 \mathrm{mmol} / 1$. $^{7}$

Coronary lesions were visually estimated in two perpendicular views and expressed as the percentage of diameter reduction as compared with the nearest "normal" arterial segments. A lesion was considered to be clinically significant when the mean value of the two views was $\geqslant 50 \%$. The characteristics of individual lesions were entered into the data bank and we used the modified American Heart Association/ American College of Cardiology scoring system to classify the lesions. ${ }^{7}$ Primary clinical success was defined as a $\geqslant 20 \%$ reduction in luminal narrowing to a residual narrowing of $<50 \%$ without the occurrence of death, myocardial infarction, or emergency surgery. Myocardial infarction was defined as an increase in cardiac enzymes (to more than twice the upper normal limits of creatine kinase and for an $M B$ fraction $>4 \%$ ).

\section{TECHNIQUE}

All percutaneous transluminal coronary angioplasty procedures were performed via the femoral or brachial approach with a rail-type device as detailed previously. ${ }^{2}$ All images were recorded by a high definition digital Polytron system (Siemens, Erlangen, Germany) that allows real time acquisition on and immediate postprocessing recall of the acquired images.

Premedication routinely consisted of aspirin $100 \mathrm{mg}$ daily, calcium antagonists, and an heparin infusion a rate of $1000 \mathrm{IU} / \mathrm{h}$. At the start of the procedure an intra-arterial bolus of heparin (10 000 IU) was given and a bolus of $5000 \mathrm{IU}$ was repeated if the procedure lasted more than an hour. After the procedure the heparin infusion was continued for 24 hours and treatment with calcium antagonists and aspirin for six months. If we could not obtain a sustained satisfactory result with the conventional balloon we introduced the Stack catheter on an extension wire. The wire was then withdrawn and positioned proximal to the sideholes of the balloon catheter in order to allow continuous perfusion during balloon inflation. After balloon inflation, the guiding catheter was pulled back carefully over a few centimetres to ensure free flow through all its sideholes. Before we removed the Stack catheter we repositioned the guiding catheter and advanced the wire into its original distal position before the final angiograms were obtained.

\section{PATIENT FOLLOW UP}

After hospital discharge repeat coronary angiography was only performed if it was judged to be clinically indicated. Every patient was scheduled for an outpatient visit and functional testing at least six months after angioplasty. Follow up data were obtained from clinical records and physician mail or telephone questionnaire.
STATISTICAL ANALYSIS

Data are expressed as mean (SD). We used the unpaired $t$ test for intergroup comparison. A $p$ value of $\leqslant 0.05$ was regarded as significant.

\section{Results}

\section{BASELINE CHARACTERISTICS}

Table 1 shows the baseline clinical characteristics of the 24 study patients. All patients underwent single lesion angioplasty. In 13 patients the attempted lesion was located in the left anterior descending artery, in seven patients it was in the right coronary artery, and in the remaining four patients it was in the left circumflex artery. A type A lesion was dilated in four patients whereas types B1 and B2 lesions were present in 10 patients each. Despite repeated attempts at redilatation with a conventional balloon catheter (mean cumulative inflation time of $418 \mathrm{~s}$ (range 1001500)) sustained vessel patency could not be achieved in any of these 24 patients. In 11 patients $(46 \%)$ recurrent thrombus formation was the most striking angiographic finding, whereas in 13 patients $(54 \%)$ a double lumen contour caused by dissection was most prominent. All 24 patients had both persisting ischaemic electrocardiographic changes and severe chest pain during and after these conventional balloon inflations.

\section{SHORT TERM OUTCOME}

The mean balloon size of the Stack catheter resembled that of the previously used balloon $(2.92(0.24) \mathrm{mm} v 2.77(0.66) \mathrm{mm} ; \mathrm{p}=\mathrm{NS})$ ) and the Stack balloon inflation time was 1728 (1316) s. In only three patients were both the clinical and electrocardiographic signs of ischaemia suppressed during the Stack balloon inflation. In three other patients only the ischaemic electrocardiographic changes disappeared and one patient became free of chest pain despite the persistence of electrocardiographic changes. In the remaining 17 patients (71\%) minor electrocardiographic changes persisted during Stack inflation while the intensity of the chest pain was sufficiently reduced to allow inflation times of many minutes. In 16 patients $(67 \%)$ a rewarding angiographic result was achieved (percentage

Table 1 Baseline characteristics of 24 patients treated with an autoperfusion catheter

\begin{tabular}{lc}
\hline Variable & Result \\
\hline Female/male ratio & $5 / 19$ \\
Age (yrs) (mean (SD)) & $57 \cdot 4(9 \cdot 3)$ \\
Cholesterol (mmol/l) (mean (SD)) & $6 \cdot 28(1 \cdot 12)$ \\
Smoking (n): & \\
Never & 5 \\
Ex & 10 \\
Current & 9 \\
Systemic hypertension (n) & 8 \\
Diabetes mellitus (n) & 5 \\
Previous myocardial infarction (n) & 12 \\
Angina class I-II (n) & 6 \\
Angina class III-IV^ (n) & 18 \\
Vessel disease (n): & 15 \\
1 & 8 \\
2 & 1 \\
3 & $69 \cdot 6(10 \cdot 9)$ \\
Ejection fraction (\%) (mean (SD))
\end{tabular}

*Canadian Cardiovascular Society classification. 
Table 2 Angiographic characteristics of the lesions successfully and unsuccessfully dilated by the Stack autoperfusion balloon catheter

\begin{tabular}{lll}
\hline Characteristic & $\begin{array}{l}\text { Successful } \\
(n=16)\end{array}$ & $\begin{array}{l}\text { Unsuccessful } \\
(n=8)\end{array}$ \\
\hline Arteries (n): & & \\
RCA & 5 & 3 \\
LAD & 2 & 4 \\
LCX & 9 & 1 \\
Subtotal and total occlusion (n) & 5 & 1 \\
Long stenotic segment (n) & 3 & 2 \\
Multiple stenoses ${ }^{\star}(\mathrm{n})$ & 1 & 0 \\
Located in a bend $>45^{\circ}(\mathrm{n})$ & 3 & 3 \\
Eccentric (n) & 8 & 6 \\
Calcified (n) & 3 & 4 \\
Presence of thrombus (n) & 4 & 3 \\
Lesion classification $\dagger(\mathrm{n}):$ & 3 & \\
A & 7 & 1 \\
B1 & 6 & 4 \\
B2 & & 4 \\
Stenosis diameter $(\%)(m e a n(S D)) 91(3)$ & $91(3)$
\end{tabular}

*Multiple stenoses within the same balloon length; †modified ACC/AHA classification system; LAD, left anterior descending artery; LCX, left circumflex artery; RCA, right coronary artery. No statistically significant differences were found between the groups.

diameter obstruction before 91 (6)\% $v 19$ (10)\% after dilatation) and in only three of these patients was a non-obstructive intimal flap visible on the final angiograms. In three of these patients there was a slight increase in creatine kinase $(<200 \mathrm{IU} / 1$, normal range 10-70 IU/1) within 24 hours. This finding was considered to be related to the occlusion of a minor side branch in one patient and to prolonged episodes of severe ischaemia during a complicated dilatation procedure in the two others. In eight $(33 \%)$ patients the Stack dilatation was unsuccessful. Emergency bypass surgery was performed in seven but the most recent failure was successfully treated with a balloon expandable stent. Table 2 gives detailed descriptions of the lesions both subgroups (successful and unsuccessful Stack dilatation).

\section{FOLLOW UP RESULTS}

The 16 patients successfully treated with the Stack autoperfusion catheter were followed up for $6 \cdot 7(2 \cdot 6)$ (range 2-11) months. Ten patients remained totally symptom free but severe (class III-IV) and mild (class I-II) anginal symptoms recurred in three patients each. Repeat coronary angiography showed restenosis in four patients and progression of disease in an undilated vessel and insignificant findings in one patient each. Two of the three patients with severe symptoms had elective surgery and in one patient repeat coronary angioplasty was performed. The three patients with minor symptoms were all treated medically.

\section{Discussion}

We found that an autoperfusion balloon catheter could be used to treat acute vessel closure after coronary angioplasty when attempted redilatation with the conventional balloon had been unsuccessful. All 16 (43\% of 37) patients in whom autoperfusion was successful would otherwise have been candidates for emergency bypass surgery. The restenosis rate after successful autoperfusion was similar to that after conventional angioplasty.
An autoperfusion catheter was first used in 1988 to perform very prolonged balloon inflations during clinical angioplasty. Stack's group used the catheter in 11 patients undergoing elective angioplasty and showed that if significantly reduced ischaemic symptoms and signs during angioplasty, thus allowing prolonged periods of balloon inflation. ${ }^{34}$ Use of the Stack coronary autoperfusion catheter was later extended to the treatment of acute vessel closure caused by large dissections during angioplasty. ${ }^{89}$ The appealing rationale for the use of the autoperfusion catheter in this setting was that the prolonged balloon inflations allowed by this catheter could be effective in "tacking up" the intimal flap."

There are only a few reports, mainly preliminary studies, about the use of this catheter system in the treatment of acute vessel closure. Saenz et $a l^{8}$ reported excellent results in 22 patients with acute occlusion or obstructive dissection during angioplasty (in 20 of them an attempt to correct the angiographic appearances with a conventional balloon had failed). A successful result was achieved with the autoperfusion catheter in all but one patient: this patient underwent coronary bypass surgery 48 hours after the procedure. Leitschuh et al reported use of the Stack autoperfusion catheter in 22 patients with coronary dissection at angioplasty. ${ }^{10}$ Angiographic success was achieved in $19(86 \%)$ : one patient $(5 \%)$ died and one patient required emergency bypass surgery. Hodes $e t$ al used the Stack catheter in two clinical settings during angioplasty. ${ }^{9}$ In 17 patients the indication for use of this catheter was treatment of dissection with repeated total or subtotal reocclusion. In four patients, all with compromised left ventricular function, the indication was the prevention of profound ischaemia during angioplasty. Success was achieved in 15 (71\%) of these 21 patients: one patient died and three required emergency coronary bypass surgery. In contrast to our study, none of the earlier, mainly preliminary, studies reported in what proportion of all their patients with acute vessel closure, not responsive to redilatation with a conventional balloon, use of a coronary autoperfusion catheter was attempted. In our series we attempted to treat refractory acute vessel closure with a Stack catheter in two thirds of the patients with this complication. In the other third, the angioplasty operator judged that the underlying dissection was too extensive for use of the autoperfusion catheter to be successful. The successful deployment of an intracoronary stent in one of the eight patients in whom we failed to achieve a successful angiographic result with the Stack catheter illustrates yet another approach to avoiding surgery in patients with acute vessel closure.

Also in contrast to our study none of the earlier studies reported follow up results after successful use of a coronary autoperfusion catheter in patients with acute vessel closure. In our study, at short-term follow up most of the patients were angina free or had only mild symptoms. In particular, the clinical restenosis rate $(25 \%)$ in our series resembles that reported 
after conventional balloon dilatation. ${ }^{2}$ The latter observation is of interest because several clinical studies have suggested that use of long inflations might reduce the restenosis rate after angioplasty. ${ }^{11}$ However, Quigley et al have recently reported the short-term follow up in 63 patients undergoing elective angioplasty with long inflations and the Stack autoperfusion catheter. ${ }^{12}$ Angiographic restenosis developed in $19(30 \%)$ of these 63 patients. This observation led Quigley et al to conclude that use of prolonged inflations did not reduce the restenosis rate. ${ }^{12}$

As with the other reports of the use of a coronary autoperfusion system in the treatment of acute vessel closure, the present study is somewhat limited by the number of patients and the retrospective nature of the study. However, our experiences were encouraging. Finally, since our centre and others are now evaluating the usefulness of newly developed balloon expandable stents as an alternative treatment for this complication, the present data may serve as reference and base for comparison.

We thank Carine Vrancken, Sabine Van Roey, Marianne Potargent, and Joke Tack for their help in preparing this paper and Stef Renkens for programming assistance.
1 Detre KM, Holmes DR, Holubkov R, et al. Incidence and consequences of periprocedural occlusions: the 1985-1986 National Heart, Lung and Blood Institute Percutaneous Transluminal Coronary Angioplasty Registry. Circulation 1990;82:739-50.

2 Stammen F, Piessens J, Vrolix M, Glazier JJ, De Geest H, Willems JL. Immediate and short term results of a 19881989 coronary angioplasty registry. Am J Cardiol 1991; 67:253-8.

3 Quigley PJ, Hinohara T, Philips HR, et al. Myocardial protection during coronary angioplasty with an autoprotection during coronary angioplasty with an auto78:1128-34.

4 Stack RS, Quigley PJ, Collins G, Phillips HR. Perfusion balloon catheter. Am J Cardiol 1988;61:133-6.

5 Willems JL, Piessens JH, De Wel M, Renkens S. Computerized reporting and information system for percutaneous transluminal coronary angioplasty procedures. In: Ripley KL, ed. Computers in cardiology. Long Beach: IEEE computer society, 1990:281-4.

6 Campeau L. Grading of angina pectoris. Circulation 1975;54:522.

7 Gotto AM, Farmer JA. Risk factors for coronary artery disease. In: Braunwald E, ed. Heart disease. A textbook of cardiovascular disease; 3rd ed. Philadelphia: WB Saunders, 1988:1153-90.

8 Saenz CB, Schwartz KM, Slysh SJ, Palenca K, Curry CR. Experience with the use of the coronary autoperfusion Experience with the use of the coronary autoperfusion Catheter during complicated

9 Hodes ZI, Rotbaum DA, Linnemeier TJ, et al. Use of the ACS Stack perfusion dilatation catheter in PTCA to avoid coronary artery bypass surgery [abstract]. J Am Coll Cardiol 1989;13:155.

10 Leitschuh ML, LaRosa D, Currier JW, et al. The "Stack" perfusion catheter improves outcome following dissection during coronary angioplasty [abstract]. J Am Coll Cardiol 1990;15:250

11 Kaltenbach M, Meyers J, Waller S, Klepzig M, Schmidts L. Prolonged application of pressure in transluminal coronary angioplasty. Cathet Cardiovasc Diagn 1984; 10:213-9.

12 Quigley PJ, Kereiakes DJ, Abbottsmith CW, et al. Prolonged autoperfusion angioplasty: immediate clinical outcome and angiographic follow-up [abstract]. J Am Coll Cardiol 1989;13:155. 